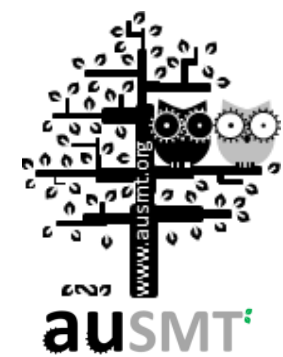

\title{
Delay-dependent Robust Stabilization for Uncertain T-S Fuzzy Systems with Additive Time Varying Delays
}

\author{
S. Idrissi1,* E. H. Tissir ${ }^{2}$, and I. Boumhidi2 \\ ${ }^{1}$ Department of Physics, Faculty of Polydisciplinary Safi, University Cadi Ayyad, BP : 4162 SAFI, Morocco \\ ${ }^{2}$ Department of Physics, Faculty of Sciences B.P. 1796 Fès-Atlas, Morocco \\ *Corresponding author: said_idrissi09@yahoo.fr \\ DOI: 10.5875 /ausmt.v7i2.1278
}

Abstract: This paper investigates the problem of robust stabilization for uncertain Takagi-Sugeno (T-S) fuzzy systems with additive time varying delays. An appropriate Lyapunov-Krasovskii function is considered for solving this problem, and obtains considerably less conservative results than existing methods. The proposed approach constructs a new Lyapunov-Krasovskii functional using two additive delay components, and no free weighting matrices are employed in the theoretical result derivation. This reduces the number of scalar decision variables in linear matrix inequalities. The fuzzy state feedback gain is derived through the numerical solution of a set of linear matrix inequalities (LMIs). Finally, numerical examples are provided to illustrate the effectiveness of the proposed method, and to allow comparison with previous works.

Keywords: additive time varying delays, T-S fuzzy control systems, liner matrix inequality (LMI), stabilization

\section{Introduction}

Over the past few decades, considerable attention has focused on fuzzy systems in the form of the TakagiSugeno (T-S) model [1]. The TS model-based control method has been shown to provide an effective way to represent some complex non-linear systems. It combines flexible fuzzy logic theory and fruitful linear system theory into a unified framework to approximate complex nonlinear systems, especially those with incomplete information, and thus has emerged as an effective way to model and control complex systems, including time delay systems. The stability issue of T-S fuzzy control systems in non-linear stability frameworks has been studied extensively, e.g. [2-4].

Time delay can introduce instability in dynamic systems and degrade system performance. This complex phenomenon appears frequently in many industrial and engineering systems, such as aircraft stabilization, manual controls, laser models, neural networks, nuclear reactors, rolling mill systems, and communication networks $[5,6]$. Recently, stability analysis and stabilizing controller design for T-S fuzzy systems with time-varying delay have

www.ausmt.org

Copyright @ 2017 International Journal of Automation and Smart Technology attracted considerable attention for use in time delays systems ([7-12]). Some proposed approaches provide stability analysis and controller synthesis of T-S fuzzy systems with time-varying delays [13-16]. For instance, in [17], the authors applied the Lyapunov-Razumikhin functional approach to stability analysis and stabilization. Tian and Peng [18] used the Lyapunov-Krasovskii functional method to investigate delay-dependent stability and controller design problems in uncertain nonlinear time-varying delay systems via T-S fuzzy models. In addition, much effort has been recently devoted to the development of the free-weighting matrix method. Efforts in $[7,8,18,19]$ theoretically applied free-weighting matrixes to investigate stability and stabilization for systems with interval time-varying delays. The methods presented in $[7,8,18,19]$ have been shown to be more effective than the previous method.

On the other hand, the TS fuzzy systems with timevarying delays have been modelled as systems with a single delay term in the state vector. More recently, the authors in [20] modeled the TS fuzzy system with two additive delay components, which have developed sufficient conditions for asymptotic stability and robust 
stability analysis by using the Lyapunov-Krasovskii functional method and Finsler's lemma. This approach produced a more effective less conservative result than was obtained in some existing methods.

This paper deals with delay-dependent stability and controller design problems for uncertain T-S fuzzy systems with two additional delay components. Sufficient stability analysis and controller design criteria are derived based on the Lyapunov-Krasovskii functional approach and Jensen's inequality. By solving a set of LMIs, the state feedback gain and the upper bound of the time delay can be obtained. We provide two illustrative examples to show that the proposed design method is less conservative.

Lemma 1 ([6], Jensen's inequality): for any constant matrix $M=M^{T} \in R^{n \times n}, M>0$, scalar $\eta>0$, vector function $\omega:[0, \eta] \rightarrow R^{n}$ such that the integrations in the following are well defined, then :

$$
\eta \int_{0}^{\eta} \omega^{\mathrm{T}}(\beta) \mathrm{M} \omega(\beta) \mathrm{d} \beta \geq\left[\int_{0}^{\eta} \omega(\beta) \mathrm{d} \beta\right]^{\mathrm{T}} \mathrm{M}\left[\int_{0}^{\eta} \omega(\beta) \mathrm{d} \beta\right]
$$

Lemma 2 [21]: Let $Q=Q^{T}, H, E$, and $F(t)$ satisfying $\mathrm{F}^{\mathrm{T}}(\mathrm{t}) \mathrm{F}(\mathrm{t}) \leq \mathrm{I}$ are appropriately dimensioned matrices, with the following inequality :

$$
\mathrm{Q}+\mathrm{HF}(\mathrm{t}) \mathrm{E}+\mathrm{E}^{\mathrm{T}} \mathrm{F}^{\mathrm{T}}(\mathrm{t}) \mathrm{H}^{\mathrm{T}}<0
$$

is true, if and only if the following inequality holds for any scalar $\varepsilon>0$

$$
\mathrm{Q}+\varepsilon^{-1} \mathrm{H} \mathrm{H}^{\mathrm{T}}+\varepsilon \mathrm{E}^{\mathrm{T}} \mathrm{E}<0
$$

\section{System description and preliminaries}

Consider the uncertain T-S fuzzy model, composed of a set of fuzzy implications, where each implication is expressed by a linear system model. The plant rule I of the

Said Idrissi received his Master in Engineering of automated industrial systems and his Ph.D. degree from University of Sidi Med Ben Abdellah in 2009 and 2014, respectively. His research interest covers stability and stabilization of T-S fuzzy systems with time delays. His current research interests include Takagi-Sugeno (T-S) fuzzy systems, robust fuzzy logic control, and time delay systems.

E-mail:Said idrissi09@yahoo.fr

El Houssaine Tissir was born in 1965. He received his Diplôme dEtudes supérieurs (DES) and Doctorat détat from the University Sidi Mohammed Ben Abellah, at Fès, Morocco in 1992 and 1997, respectively. He has been with the Department of Physics, in the same university, Dhar El Mehraz at Fes, where he is currently a Full Professor. His current research interests include robust control, time delay systems, systems with saturating actuators, $\mathrm{H} 1$ control, singular systems and neutral systems.

E-mail:elh tissir@yahoo.fr

Ismail Boumhidi is a Professor of electronics at the Faculty of Sciences, Fez, Morocco. He received his Ph.D. degree from Sidi Mohamed ben Abdellah University, Faculty of sciences, in 1999. His research areas include adaptive robust control, multivariable nonlinear systems, and fuzzy logic control with applications.

E-mail:iboumhidi@hotmail.com
T-S fuzzy model is described by the following IF - THEN statement:

Plant Rule i:

IF $\mathrm{z}_{1}(\mathrm{t})$ is $\mathrm{W}_{1}^{\mathrm{i}}$ and $\ldots$ and $\mathrm{z}_{\mathrm{g}}(\mathrm{t})$ is $\mathrm{W}_{\mathrm{g}}^{\mathrm{i}}$ THEN

$\left\{\begin{aligned} \dot{\mathrm{x}}(\mathrm{t}) & =\left(\mathrm{A}_{0 \mathrm{i}}+\Delta \mathrm{A}_{0 \mathrm{i}}(\mathrm{t})\right) \mathrm{x}(\mathrm{t})+\left(\mathrm{A}_{\mathrm{di}}+\Delta \mathrm{A}_{\mathrm{di}}(\mathrm{t})\right) \mathrm{x}\left(\mathrm{t}-\mathrm{h}_{1}(\mathrm{t})-\mathrm{h}_{2}(\mathrm{t})\right) \\ & +\left(\mathrm{B}_{\mathrm{i}}+\Delta \mathrm{B}_{\mathrm{i}}(\mathrm{t})\right) \mathrm{u}(\mathrm{t}) \\ \mathrm{x}(\mathrm{t}) & =\phi(\mathrm{t}), \mathrm{t} \in[-\overline{\mathrm{h}}, 0], \quad \mathrm{i}=1,2, \ldots, \mathrm{r}\end{aligned}\right.$

where $\mathrm{z}_{1}(\mathrm{t}), \mathrm{z}_{2}(\mathrm{t}), \ldots, \mathrm{z}_{\mathrm{g}}(\mathrm{t})$ are the premise variables, $\mathrm{W}_{\mathrm{j}}^{\mathrm{i}}, \mathrm{j}=1,2, \ldots, \mathrm{g}$ are fuzzy sets, $\mathrm{x}(\mathrm{t}) \in \mathrm{R}^{\mathrm{n}}$ is the state variable, and $\mathrm{u}(\mathrm{t}) \in \mathrm{R}^{\mathrm{m}}$ is the control input. $\mathrm{A}_{0 \mathrm{i}}, \mathrm{A}_{\mathrm{di}} \in \mathrm{R}^{\mathrm{n} \times \mathrm{n}}, \mathrm{B}_{\mathrm{i}} \in \mathrm{R}^{\mathrm{n} \times \mathrm{m}}, \mathrm{r}$ is the number of if-then rules, $\phi(\mathrm{t})$ is a vector-valued initial condition of systems $(1)$, and $\mathrm{h}_{1}(\mathrm{t})$ and $h_{2}(t)$ are the time-varying delays satisfying

$$
\begin{gathered}
0 \leq h_{1}(t) \leq \bar{h}_{1}, \dot{\mathrm{h}}_{1}(\mathrm{t}) \leq \mathrm{d}_{1}, 0 \leq \mathrm{h}_{2}(\mathrm{t}) \leq \overline{\mathrm{h}}_{2}, \quad \dot{\mathrm{h}}_{2}(\mathrm{t}) \leq \mathrm{d}_{2} ， \\
\overline{\mathrm{h}}=\overline{\mathrm{h}}_{1}+\overline{\mathrm{h}}_{2} \text { and } \mathrm{d}=\mathrm{d}_{1}+\mathrm{d}_{2}
\end{gathered}
$$

The parametric uncertainties $\Delta \mathrm{A}_{0 \mathrm{i}}(\mathrm{t}), \Delta \mathrm{A}_{\mathrm{di}}(\mathrm{t})$ and $\Delta \mathrm{B}_{\mathrm{i}}(\mathrm{t})$ are time-varying matrices with appropriate dimensions, which can be described as:

$$
\begin{array}{cl}
{\left[\begin{array}{ll}
\Delta \mathrm{A}_{0 \mathrm{i}}(\mathrm{t}) \quad & \Delta \mathrm{A}_{\mathrm{di}}(\mathrm{t}) \quad \Delta \mathrm{B}_{\mathrm{i}}(\mathrm{t})
\end{array}\right]=\mathrm{D}_{\mathrm{i}} \mathrm{F}_{\mathrm{i}}(\mathrm{t})\left[\begin{array}{lll}
\mathrm{E}_{0 \mathrm{i}} & \mathrm{E}_{\mathrm{di}} & \mathrm{E}_{\mathrm{bi}}
\end{array}\right],} \\
\mathrm{i}=1,2, \ldots, \mathrm{r}
\end{array}
$$

where $\mathrm{D}_{\mathrm{i}}, \mathrm{E}_{0 \mathrm{i}}, \mathrm{E}_{\mathrm{di}}$ and $\mathrm{E}_{\mathrm{bi}}$ are known constant real matrices with appropriate dimensions and $\mathrm{F}_{\mathrm{i}}(\mathrm{t})$ are unknown real time-varying matrices with Lebesgue measurable elements bounded by:

$$
F_{i}^{T}(t) F_{i}(t) \leq I, i=1,2, \ldots, r
$$

For simplicity, we introduce the following notations: $\overline{\mathrm{A}}_{0 \mathrm{i}}=\mathrm{A}_{0 \mathrm{i}}+\Delta \mathrm{A}_{0 \mathrm{i}}(\mathrm{t}), \overline{\mathrm{A}}_{\mathrm{di}}=\mathrm{A}_{\mathrm{di}}+\Delta \mathrm{A}_{\mathrm{di}}(\mathrm{t})$

and $\bar{B}_{i}=B_{i}+\Delta B_{i}(t)$

By using the center-average deffuzzifier, product inference and singleton fuzzifier, the global dynamics of T$Z$ fuzzy system (1) can be expressed as

$$
\dot{x}(t)=\sum_{i=1}^{r} \mu_{i}(z(t))\left[\bar{A}_{0 i} x(t)+\bar{A}_{d i} x\left(t-h_{1}(t)-h_{2}(t)\right)+\bar{B}_{i} u(t)\right]
$$

where, $\quad \mu_{\mathrm{i}}(\mathrm{z}(\mathrm{t}))=\omega_{\mathrm{i}}(\mathrm{z}(\mathrm{t})) / \sum^{\mathrm{r}} \omega_{\mathrm{i}}(\mathrm{z}(\mathrm{t}))$

$\omega_{i}(z(t))=\prod^{\mathrm{g}} \mathrm{W}_{\mathrm{j}}^{\mathrm{i}}\left(\mathrm{z}_{\mathrm{j}}(\mathrm{t})\right) \mathrm{z}(\mathrm{t})=\left[\mathrm{z}_{1}(\mathrm{t}), \ldots, \mathrm{z}_{\mathrm{g}}(\mathrm{t})\right]^{\mathrm{T}} \quad$ and $\mathrm{W}_{\mathrm{j}}^{\mathrm{i}}\left(\mathrm{z}_{\mathrm{j}}(\mathrm{t})\right)$ is the membership value of $\mathrm{z}_{j}(\mathrm{t})$ in $\mathrm{W}_{\mathrm{j}}^{\mathrm{i}}$. Some basic properties of $\mu_{\mathrm{i}}(\mathrm{z}(\mathrm{t}))$ are $\mu_{\mathrm{i}}(\mathrm{z}(\mathrm{t})) \geq 0$, $\sum_{i=1}^{\mathrm{r}} \mu_{\mathrm{i}}(\mathrm{z}(\mathrm{t}))=1$.

$\sum_{i=1}$ The ith controller rule is:

Control Rule i: 
IF $\mathrm{z}_{1}(\mathrm{t})$ is $\mathrm{W}_{1}^{\mathrm{i}}$ and $\ldots$ and $\mathrm{z}_{\mathrm{g}}(\mathrm{t})$ is $\mathrm{W}_{\mathrm{g}}^{\mathrm{i}}$

Then $\mathrm{u}(\mathrm{t})=\mathrm{K}_{\mathrm{i}} \mathrm{x}(\mathrm{t}), \quad \mathrm{i}=1,2, \ldots, \mathrm{r}$,

The defuzzified output of controller rule (6) is proposed as

$$
u(t)=\sum_{j=1}^{r} \mu_{j}(z(t)) K_{j} x(t)
$$

where $\mathrm{K}_{\mathrm{j}}(\mathrm{j}=1,2, \ldots, \mathrm{r})$ are the local controller gains to be determined. The fuzzy controller is designed to determine the feedback gains $\mathrm{K}_{\mathrm{j}}(\mathrm{j}=1,2, \ldots, \mathrm{r})$ such that the closed-loop system is asymptotically stable.

Combining (5) and (7), we obtain the following closed-loop fuzzy system:

$$
\left\{\begin{array}{c}
\dot{\mathrm{x}}(\mathrm{t})=\sum_{\mathrm{i}=1}^{\mathrm{r}} \sum_{\mathrm{j}=1}^{\mathrm{r}} \mu_{\mathrm{i}}(\mathrm{z}(\mathrm{t})) \mu_{\mathrm{j}}(\mathrm{z}(\mathrm{t}))\left[\left(\overline{\mathrm{A}}_{0 \mathrm{i}}+\overline{\mathrm{B}}_{\mathrm{i}} \mathrm{K}_{\mathrm{j}}\right) \mathrm{x}(\mathrm{t})+\overline{\mathrm{A}}_{\mathrm{di}} \mathrm{x}\left(\mathrm{t}-\mathrm{h}_{1}(\mathrm{t})-\mathrm{h}_{2}(\mathrm{t})\right)\right] \\
\mathrm{x}(\mathrm{t})=\phi(\mathrm{t}), \mathrm{t} \in\left[\begin{array}{ll}
-\overline{\mathrm{h}} & 0
\end{array}\right], \mathrm{i}=1,2, \ldots, \mathrm{r}
\end{array}\right.
$$

Remark 1: The time delay verifying (2) represents the real situation in many practical applications. For example, in networked controlled systems successive delays with different properties are introduced into signal transmission, either from sensor to controller $h_{1}(t)$ or from controller to actuator $h_{2}(t)$, and these delays are actually timevarying. The stability analysis of such system has intervened out by adding up all the successive delays, i.e., $\mathrm{h}(\mathrm{t})=\mathrm{h}_{1}(\mathrm{t})+\mathrm{h}_{2}(\mathrm{t})$. However, to the best of our knowledge, few papers have considered the additive timevarying delay case for T-S fuzzy systems.

\section{Robust stability analysis and synthesis}

This section analyzes the stability of closed-loop T-S fuzzy time-varying systems with two additive time varying delays (8), based on a new Lyapunov-Krasovskii functional approach.

Theorem 1. For given scalars $\overline{\mathrm{h}}_{1} \geq 0, \overline{\mathrm{h}}_{2} \geq 0$ and matrices $K_{j}$, system (8) satisfying conditions (2) is robustly asymptotically stable, if there exist symmetric positive definite matrices $\mathrm{P}, \mathrm{Q}_{1}, \mathrm{Q}_{2}, \mathrm{Z}_{1}$ and $\mathrm{Z}_{2}$ with appropriate dimensions, and scalar $\varepsilon_{\mathrm{ij}}>0$ such that $\mathrm{Q}_{1}-\mathrm{Q}_{2} \geq 0$ and the following linear matrix inequalities (LMIs) hold for $\mathrm{i}, \mathrm{j}=1,2, \ldots, \mathrm{r}$,

$$
\Sigma_{\mathrm{ij}}=\left[\begin{array}{cccccc}
\Sigma_{11}^{\mathrm{ij}} & \Sigma_{12}^{\mathrm{ij}} & \Sigma_{13}^{\mathrm{ij}} & \Sigma_{14}^{\mathrm{ij}} & \Sigma_{15}^{\mathrm{ij}} & \Sigma_{16}^{\mathrm{ij}} \\
* & \Sigma_{22}^{\mathrm{ij}} & \Sigma_{23}^{\mathrm{ij}} & 0 & 0 & 0 \\
* & * & \Sigma_{33}^{\mathrm{ij}} & \Sigma_{34}^{\mathrm{ij}} & 0 & \Sigma_{36}^{\mathrm{ij}} \\
* & * & * & \Sigma_{44}^{\mathrm{ij}} & \Sigma_{45}^{\mathrm{ij}} & 0 \\
* & * & * & * & \Sigma_{55}^{\mathrm{ij}} & 0 \\
* & * & * & * & * & \Sigma_{66}^{\mathrm{ij}}
\end{array}\right]<0
$$

where,

$\Sigma_{11}^{\mathrm{ij}}=\mathrm{P}\left(\mathrm{A}_{0 \mathrm{i}}+\mathrm{B}_{\mathrm{i}} \mathrm{k}_{\mathrm{j}}\right)+\left(\mathrm{A}_{0 \mathrm{i}}+\mathrm{B}_{\mathrm{i}} \mathrm{k}_{\mathrm{j}}\right)^{\mathrm{T}} \mathrm{P}-\mathrm{Z}_{1}+\mathrm{Q}_{1}$,

$\Sigma_{12}^{\mathrm{ij}}=\mathrm{Z}_{1}, \quad \Sigma_{13}^{\mathrm{ij}}=\mathrm{PA}_{\mathrm{di}}, \quad \Sigma_{14}^{\mathrm{ij}}=\left(\mathrm{A}_{0 \mathrm{i}}+\mathrm{B}_{\mathrm{i}} \mathrm{K}_{\mathrm{j}}\right)^{\mathrm{T}} \mathrm{P}$,

$\Sigma_{15}^{\mathrm{ij}}=\mathrm{PD}_{\mathrm{i}}, \Sigma_{16}^{\mathrm{ij}}=\varepsilon_{\mathrm{ij}}\left(\mathrm{E}_{0 \mathrm{i}}+\mathrm{E}_{\mathrm{bi}} \mathrm{K}_{\mathrm{j}}\right)^{\mathrm{T}}$

$\Sigma_{22}^{\mathrm{ij}}=-\mathrm{Z}_{1}-\mathrm{Z}_{2}-\left(1-\mathrm{d}_{1}\right)\left(\mathrm{Q}_{1}-\mathrm{Q}_{2}\right), \Sigma_{23}^{\mathrm{ij}}=\mathrm{Z}_{2}$

$\Sigma_{33}^{\mathrm{ij}}=-\mathrm{Z}_{2}-\left(1-\mathrm{d}_{1}-\mathrm{d}_{2}\right) \mathrm{Q}_{2}, \Sigma_{34}^{\mathrm{ij}}=\mathrm{A}_{\mathrm{di}}^{\mathrm{T}} \mathrm{P}, \Sigma_{36}^{\mathrm{ij}}=\varepsilon_{\mathrm{ij}} \mathrm{E}_{\mathrm{di}}^{\mathrm{T}}$

$\Sigma_{44}^{\mathrm{ij}}=\mathrm{h}_{1}^{2} \mathrm{Z}_{1}+\mathrm{h}_{2}^{2} \mathrm{Z}_{2}-2 \mathrm{P}, \Sigma_{45}^{\mathrm{ij}}=\mathrm{PD}_{\mathrm{i}}$

$\Sigma_{55}^{\mathrm{ij}}=-\varepsilon_{\mathrm{ij}} \mathrm{I}, \quad \Sigma_{66}^{\mathrm{ij}}=-\varepsilon_{\mathrm{ij}} \mathrm{I}$

Proof: Define the following Lyapunov-Krasovskii functional

$$
\mathrm{V}\left(\mathrm{x}_{\mathrm{t}}\right)=\mathrm{V}_{1}\left(\mathrm{x}_{\mathrm{t}}\right)+\mathrm{V}_{2}\left(\mathrm{x}_{\mathrm{t}}\right)+\mathrm{V}_{3}\left(\mathrm{x}_{\mathrm{t}}\right)
$$

where,

$$
\begin{aligned}
& \mathrm{V}_{1}\left(\mathrm{x}_{\mathrm{t}}\right)=\mathrm{x}^{\mathrm{T}}(\mathrm{t}) \operatorname{Px}(\mathrm{t}) \\
& \mathrm{V}_{2}\left(\mathrm{x}_{\mathrm{t}}\right)=\overline{\mathrm{h}}_{1} \int_{-\overline{\mathrm{h}}_{1}}^{0} \int_{\mathrm{t}+\theta}^{\mathrm{t}} \dot{\mathrm{x}}^{\mathrm{T}}(\mathrm{s}) \mathrm{Z}_{1} \dot{\mathrm{x}}(\mathrm{s}) \mathrm{dsd} \theta \\
& +\overline{\mathrm{h}}_{2} \int_{-\overline{\mathrm{h}}_{1}-\overline{\mathrm{h}}_{2}}^{-\overline{\mathrm{h}}_{1}} \int_{\mathrm{t}+\theta}^{\mathrm{t}} \dot{\mathrm{x}}^{\mathrm{T}}(\mathrm{s}) \mathrm{Z}_{2} \dot{\mathrm{x}}(\mathrm{s}) \mathrm{d} \mathrm{d} d \theta
\end{aligned}
$$

$V_{3}\left(x_{t}\right)=\int_{t-h_{1}(t)}^{t} x^{T}(s) Q_{1} x(s) d s+\int_{t-h_{1}(t)-h_{2}(t)}^{t-h_{1}(t)} x^{T}(s) Q_{2} x(s) d s$

computes the time derivative of $\mathrm{V}\left(\mathrm{x}_{\mathrm{t}}\right)$ along the trajectory of Eq. (8) to obtain,

$$
\begin{array}{r}
\dot{\mathrm{V}}_{1}\left(\mathrm{x}_{\mathrm{t}}\right)=2 \mathrm{x}^{\mathrm{T}}(\mathrm{t}) \mathrm{P} \times\left\{\sum _ { \mathrm { i } = 1 } ^ { \mathrm { r } } \sum _ { \mathrm { j } = 1 } ^ { \mathrm { r } } \mu _ { \mathrm { i } } ( \mathrm { z } ( \mathrm { t } ) ) \mu _ { \mathrm { j } } ( \mathrm { z } ( \mathrm { t } ) ) \left[\left(\overline{\mathrm{A}}_{0 \mathrm{i}}+\overline{\mathrm{B}}_{\mathrm{i}} \mathrm{K}_{\mathrm{j}}\right) \mathrm{x}(\mathrm{t})+\right.\right. \\
\left.\left.\overline{\mathrm{A}}_{\mathrm{di}} \mathrm{x}\left(\mathrm{t}-\mathrm{h}_{1}(\mathrm{t})-\mathrm{h}_{2}(\mathrm{t})\right)\right]\right\}
\end{array}
$$

$$
\begin{array}{r}
\dot{\mathrm{V}}_{2}\left(\mathrm{x}_{\mathrm{t}}\right)=\overline{\mathrm{h}}_{1} \int_{-\overline{\mathrm{h}}_{1}}^{0}\left[\dot{\mathrm{x}}^{\mathrm{T}}(\mathrm{t}) \mathrm{Z}_{1} \dot{\mathrm{x}}(\mathrm{t})-\dot{\mathrm{x}}^{\mathrm{T}}(\mathrm{t}+\theta) \mathrm{Z}_{1} \dot{\mathrm{x}}(\mathrm{t}+\theta)\right] d \theta+ \\
\overline{\mathrm{h}}_{2} \int_{-\overline{\mathrm{h}}_{1}-\overline{\mathrm{h}}_{2}}^{-\overline{\mathrm{h}}_{1}}\left[\dot{\mathrm{x}}^{\mathrm{T}}(\mathrm{t}) \mathrm{Z}_{2} \dot{\mathrm{x}}(\mathrm{t})-\dot{\mathrm{x}}^{\mathrm{T}}(\mathrm{t}+\theta) \mathrm{Z}_{2} \dot{\mathrm{x}}(\mathrm{t}+\theta)\right] d \theta \\
\dot{\mathrm{V}}_{2}\left(\mathrm{x}_{\mathrm{t}}\right)=\overline{\mathrm{h}}_{1}^{2} \dot{\mathrm{x}}^{\mathrm{T}}(\mathrm{t}) \mathrm{Z}_{1} \dot{\mathrm{x}}(\mathrm{t})-\overline{\mathrm{h}}_{1} \int_{\mathrm{t}-\overline{\mathrm{h}}_{1}}^{\mathrm{t}} \dot{\mathrm{x}}^{\mathrm{T}}(\mathrm{s}) \mathrm{Z}_{1} \dot{\mathrm{x}}(\mathrm{s}) \mathrm{ds}+ \\
\overline{\mathrm{h}}_{2}^{2} \dot{\mathrm{x}}^{\mathrm{T}}(\mathrm{t}) \mathrm{Z}_{2} \dot{\mathrm{x}}(\mathrm{t})-\overline{\mathrm{h}}_{2} \int_{\mathrm{t}-\overline{\mathrm{h}}^{\mathrm{t}-\overline{\mathrm{h}}_{1}} \dot{\mathrm{x}}^{\mathrm{T}}(\mathrm{s}) \mathrm{Z}_{2} \dot{\mathrm{x}}(\mathrm{s}) \mathrm{ds}}
\end{array}
$$

$\mathrm{Z}_{1}$ and $\mathrm{Z}_{2}$ are symmetric positive definite matrices, and the following inequalities always hold [22]:

$$
-\int_{\mathrm{t}-\overline{\mathrm{h}}_{1}}^{\mathrm{t}} \dot{\mathrm{x}}^{\mathrm{T}}(\mathrm{s}) \mathrm{Z}_{1} \dot{\mathrm{x}}(\mathrm{s}) \mathrm{d} \mathrm{s} \leq-\int_{\mathrm{t}-\mathrm{h}_{1}(\mathrm{t})}^{\mathrm{t}} \dot{\mathrm{x}}^{\mathrm{T}}(\mathrm{s}) \mathrm{Z}_{1} \dot{\mathrm{x}}(\mathrm{s}) \mathrm{d} s
$$




$$
-\int_{\mathrm{t}-\overline{\mathrm{h}}}^{\mathrm{t}-\overline{\mathrm{h}}_{1}} \dot{\mathrm{x}}^{\mathrm{T}}(\mathrm{s}) \mathrm{Z}_{2} \dot{\mathrm{X}}(\mathrm{s}) \mathrm{d} \mathrm{s} \leq-\int_{\mathrm{t}-\mathrm{h}(\mathrm{t})}^{\mathrm{t}-\mathrm{h}_{1}(\mathrm{t})} \dot{\mathrm{x}}^{\mathrm{T}}(\mathrm{s}) \mathrm{Z}_{2} \dot{\mathrm{x}}(\mathrm{s}) \mathrm{d} s
$$

where $\mathrm{h}(\mathrm{t})=\mathrm{h}_{1}(\mathrm{t})+\mathrm{h}_{2}(\mathrm{t})$. Using the above inequalities in (15) we have:

$$
\begin{aligned}
& \dot{\mathrm{V}}_{2}\left(\mathrm{x}_{\mathrm{t}}\right) \leq \overline{\mathrm{h}}_{1}^{2} \dot{\mathrm{x}}^{\mathrm{T}}(\mathrm{t}) \mathrm{Z}_{1} \dot{\mathrm{x}}(\mathrm{t})-\overline{\mathrm{h}}_{1} \int_{\mathrm{t}-\mathrm{h}_{1}(\mathrm{t})}^{\mathrm{t}} \dot{\mathrm{x}}^{\mathrm{T}}(\mathrm{s}) \mathrm{Z}_{1} \dot{\mathrm{x}}(\mathrm{s}) \mathrm{ds}+ \\
& \overline{\mathrm{h}}_{2}^{2} \dot{\mathrm{x}}^{\mathrm{T}}(\mathrm{t}) \mathrm{Z}_{2} \dot{\mathrm{x}}(\mathrm{t})-\overline{\mathrm{h}}_{2} \int_{\mathrm{t}-\mathrm{h}(\mathrm{t})}^{\mathrm{t}-\mathrm{h}_{1}(\mathrm{t})} \dot{\mathrm{x}}^{\mathrm{T}}(\mathrm{s}) \mathrm{Z}_{2} \dot{\mathrm{x}}(\mathrm{s}) \mathrm{ds}
\end{aligned}
$$

By using lemma 1 we obtain:

$$
\begin{aligned}
& \dot{\mathrm{V}}_{2}\left(\mathrm{x}_{\mathrm{t}}\right) \leq \overline{\mathrm{h}}_{1}^{2} \dot{\mathrm{x}}^{\mathrm{T}}(\mathrm{t}) \mathrm{Z}_{1} \dot{\mathrm{x}}(\mathrm{t})-\left[\mathrm{x}(\mathrm{t})-\mathrm{x}\left(\mathrm{t}-\mathrm{h}_{1}(\mathrm{t})\right]^{\mathrm{T}} \mathrm{Z}_{1}\left[\mathrm{x}(\mathrm{t})-\mathrm{x}\left(\mathrm{t}-\mathrm{h}_{1}(\mathrm{t})\right]+\right.\right. \\
& \overline{\mathrm{h}}_{2}^{2} \dot{\mathrm{x}}^{\mathrm{T}}(\mathrm{t}) \mathrm{Z}_{2} \dot{\mathrm{x}}(\mathrm{t})-\left[\mathrm{x}\left(\mathrm{t}-\mathrm{h}_{1}(\mathrm{t})\right)-\mathrm{x}(\mathrm{t}-\mathrm{h}(\mathrm{t})]^{\mathrm{T}} \mathrm{Z}_{2}\left[\mathrm{x}\left(\mathrm{t}-\mathrm{h}_{1}(\mathrm{t})\right)-\mathrm{x}(\mathrm{t}-\mathrm{h}(\mathrm{t})]\right.\right.
\end{aligned}
$$

$\dot{\mathrm{V}}_{3}\left(\mathrm{x}_{\mathrm{t}}\right)=\mathrm{x}^{\mathrm{T}}(\mathrm{t}) \mathrm{Q}_{1} \mathrm{x}(\mathrm{t})-\left(1-\dot{\mathrm{h}}_{1}(\mathrm{t})\right) \mathrm{x}^{\mathrm{T}}\left(\mathrm{t}-\mathrm{h}_{1}(\mathrm{t})\right) \mathrm{Q}_{1} \mathrm{x}\left(\mathrm{t}-\mathrm{h}_{1}(\mathrm{t})\right)$

$+\left(1-\dot{\mathrm{h}}_{1}(\mathrm{t})\right) \mathrm{x}^{\mathrm{T}}\left(\mathrm{t}-\mathrm{h}_{1}(\mathrm{t})\right) \mathrm{Q}_{2} \mathrm{x}\left(\mathrm{t}-\mathrm{h}_{1}(\mathrm{t})\right)-\left(1-\dot{\mathrm{h}}_{1}(\mathrm{t})-\dot{\mathrm{h}}_{2}(\mathrm{t})\right)$

$x^{T}(t-h(t)) Q_{2} x(t-h(t))$

$\dot{V}_{3}\left(x_{t}\right) \leq x^{T}(t) Q_{1} x(t)-\left(1-d_{1}\right) x^{T}\left(t-h_{1}(t)\right)\left(Q_{1}-Q_{2}\right) x\left(t-h_{1}(t)\right)$

$-\left(1-d_{1}-d_{2}\right) x^{T}(t-h(t)) Q_{2} x(t-h(t))$

where $\mathrm{Q}_{1}-\mathrm{Q}_{2} \geq 0$

Now let,

$$
\xi^{\mathrm{T}}(\mathrm{t})=\left[\begin{array}{llll}
\mathrm{x}^{\mathrm{T}}(\mathrm{t}) & \mathrm{x}^{\mathrm{T}}\left(\mathrm{t}-\mathrm{h}_{1}(\mathrm{t})\right) \quad \mathrm{x}^{\mathrm{T}}(\mathrm{t}-\mathrm{h}(\mathrm{t})) \quad \dot{\mathrm{x}}^{\mathrm{T}}(\mathrm{t})
\end{array}\right]
$$

Taking account of (14), (17) and (18), along with the following result

$$
-2 \dot{x}^{T}(t) P \dot{x}(t)+2 \dot{x}^{T}(t) P \times\left\{\begin{array}{l}
\sum_{i=1}^{r} \sum_{j=1}^{r} \mu_{i}(z(t)) \mu_{j}(z(t))\left[\left(\bar{A}_{0 i}+\bar{B}_{i} K_{j}\right) x(t)+\right. \\
\bar{A}_{d i} x\left(t-h_{1}(t)-h_{2}(t)\right)
\end{array}\right\}=0
$$

We thus obtain

$$
\dot{\mathrm{V}}\left(\mathrm{x}_{\mathrm{t}}\right) \leq \sum_{\mathrm{i}=1}^{\mathrm{r}} \sum_{\mathrm{j}=1}^{\mathrm{r}} \mu_{\mathrm{i}}(\mathrm{z}(\mathrm{t})) \mu_{\mathrm{j}}(\mathrm{z}(\mathrm{t})) \xi^{\mathrm{T}}(\mathrm{t}) \Pi_{\mathrm{ij}} \xi(\mathrm{t})
$$

where

$$
\Pi_{\mathrm{ij}}=\left[\begin{array}{cccc}
\Pi_{11}^{\mathrm{ij}} & \Sigma_{12}^{\mathrm{ij}} & \Pi_{13}^{\mathrm{ij}} & \Pi_{14}^{\mathrm{ij}} \\
* & \Sigma_{22}^{\mathrm{ij}} & \Sigma_{23}^{\mathrm{ij}} & 0 \\
* & * & \Sigma_{33}^{\mathrm{ij}} & \Pi_{34}^{\mathrm{ij}} \\
* & * & * & \Sigma_{44}^{\mathrm{ij}}
\end{array}\right]
$$

$\Pi_{11}^{\mathrm{ij}}=\mathrm{P}\left(\overline{\mathrm{A}}_{0 \mathrm{i}}+\overline{\mathrm{B}}_{\mathrm{i}} \mathrm{K}_{\mathrm{j}}\right)+\left(\overline{\mathrm{A}}_{0 \mathrm{i}}+\overline{\mathrm{B}}_{\mathrm{i}} \mathrm{K}_{\mathrm{j}}\right)^{\mathrm{T}} \mathrm{P}-\mathrm{Z}_{1}+\mathrm{Q}_{1}$

$\Pi_{13}^{\mathrm{ij}}=\mathrm{PA}_{\mathrm{di}}, \Pi_{14}^{\mathrm{ij}}=\left(\overline{\mathrm{A}}_{0 \mathrm{i}}+\overline{\mathrm{B}}_{\mathrm{i}} \mathrm{K}_{\mathrm{j}}\right)^{\mathrm{T}} \mathrm{P}, \Pi_{34}^{\mathrm{ij}}=\overline{\mathrm{A}}_{\mathrm{di}}^{\mathrm{T}} \mathrm{P}$ and $\Sigma_{12}^{\mathrm{ij}}, \Sigma_{22}^{\mathrm{ij}}, \Sigma_{23}^{\mathrm{ij}}, \Sigma_{33}^{\mathrm{ij}}, \Sigma_{44}^{\mathrm{ij}}$ are defined in (9).

Decomposing the resulting matrix $\Pi_{\mathrm{ij}}$ into nominal and uncertain parts leads to

$$
\Pi_{i j}=\Xi_{i j}+H_{i} F_{i}(t) E_{i j}+E_{i j}^{T} F_{i}^{T}(t) H_{i}^{T}
$$

where,

$$
\begin{gathered}
\Xi_{\mathrm{ij}}=\left[\begin{array}{cccc}
\Sigma_{11}^{\mathrm{ij}} & \Sigma_{12}^{\mathrm{ij}} & \Sigma_{13}^{\mathrm{ij}} & \Sigma_{14}^{\mathrm{ij}} \\
* & \Sigma_{22}^{\mathrm{ij}} & \Sigma_{23}^{\mathrm{ij}} & 0 \\
* & * & \Sigma_{33}^{\mathrm{ij}} & \Sigma_{34}^{\mathrm{ij}} \\
* & * & * & \Sigma_{44}^{\mathrm{ij}}
\end{array}\right], \\
\mathrm{H}=\left[\begin{array}{llll}
\mathrm{D}_{\mathrm{i}}^{\mathrm{T}} \mathrm{P} & 0 & 0 & \mathrm{D}_{\mathrm{i}}^{\mathrm{T}} \mathrm{P}
\end{array}\right]^{\mathrm{T}}, \\
\mathrm{E}_{\mathrm{ij}}=\left[\begin{array}{llll}
\left(\mathrm{E}_{0 \mathrm{i}}+\mathrm{E}_{\mathrm{bi}}\right) & 0 & \mathrm{E}_{\mathrm{di}} & 0
\end{array}\right]
\end{gathered}
$$

Applying Lemma 2, it is clear that the following inequalities always hold

$$
\Pi_{\mathrm{ij}}=\Xi_{\mathrm{ij}}+\mathrm{H}_{\mathrm{i}} \mathrm{F}_{\mathrm{i}}(\mathrm{t}) \mathrm{E}_{\mathrm{ij}}+\mathrm{E}_{\mathrm{ij}}^{\mathrm{T}} \mathrm{F}_{\mathrm{i}}^{\mathrm{T}}(\mathrm{t}) \mathrm{H}_{\mathrm{i}}^{\mathrm{T}}<\Xi_{\mathrm{ij}}+\mathrm{H}_{\mathrm{i}} \varepsilon_{\mathrm{ij}}^{-1} \mathrm{H}_{\mathrm{i}}^{\mathrm{T}}+\mathrm{E}_{\mathrm{ij}}^{\mathrm{T}} \varepsilon_{\mathrm{ij}} \mathrm{E}_{\mathrm{ij}}
$$

Using Schur's complement of (21), LMI (9) is equivalent to $\Pi_{\mathrm{ij}}$ in (19), then $\dot{\mathrm{V}}\left(\mathrm{x}_{\mathrm{t}}\right)<0$. Thus, system (8) is robustly asymptotically stable and the proof is complete. Remark 2: The authors in [20] proposed delay-dependent stability criteria for $\mathrm{T} S$ fuzzy systems with additive timevarying delay, in which free weighting matrices are introduced and less conservative results are obtained as compared with some existing results $[16,18,23]$. The approach developed in the present paper is based on the Lyapunov Krasovskii functional method without any free weighting matrices. Theorem 1 of this paper and theorem 1 of [20] respectively require $\frac{5 n(n+1)}{2}$ and $3 n^{2}+\frac{5 n(n+1)}{2}$ variables. Thus, theorem 1 requires fewer variables than $3 n^{2}$.Therefore, our results alleviate the computational demand required to obtain a solution for stable conditions. This advantage is especially apparent in systems with a large dimension n. Remark 3: In lemma 1, the proposed approach accounts for useful terms in the derivative of Lyapunov-Krasovskii functional, e.g. $\int_{\mathrm{t}-\overline{\mathrm{h}}_{1}}^{\mathrm{t}} \dot{\mathrm{x}}^{\mathrm{T}}(\mathrm{s}) \mathrm{Z}_{1} \dot{\mathrm{x}}(\mathrm{s}) \mathrm{ds}$ and $\int_{\mathrm{t}-\overline{\mathrm{h}}}^{\mathrm{t}-\overline{\mathrm{h}}_{1}} \dot{\mathrm{x}}^{\mathrm{T}}(\mathrm{s}) \mathrm{Z}_{2} \dot{\mathrm{x}}(\mathrm{s}) \mathrm{ds}$ in (15). Some previous studies $[17,24,25]$ estimate the derivative of $\int_{-\bar{h}_{1}}^{0} \int_{t+\theta}^{\mathrm{t}} \dot{\mathrm{x}}^{\mathrm{T}}(\mathrm{s}) \mathrm{Z}_{1} \dot{\mathrm{x}}(\mathrm{s}) \mathrm{d} \mathrm{d} \mathrm{d} \theta$

$\dot{\mathrm{x}}^{\mathrm{T}}(\mathrm{t}) \mathrm{Z}_{1} \dot{\mathrm{x}}(\mathrm{t})-\overline{\mathrm{h}}_{1} \int_{\mathrm{t}-\overline{\mathrm{h}}_{1}}^{\mathrm{t}} \dot{\mathrm{x}}^{\mathrm{T}}(\mathrm{s}) \mathrm{Z}_{1} \dot{\mathrm{x}}(\mathrm{s}) \mathrm{ds} \quad$ and $\quad$ the term $-\int_{\mathrm{t}-\overline{\mathrm{h}}_{1}}^{\mathrm{t}} \dot{\mathrm{x}}^{\mathrm{T}}(\mathrm{s}) \mathrm{Z}_{1} \dot{\mathrm{x}}(\mathrm{s}) \mathrm{ds}$ is neglected, which may lead to considerable conservativeness. As shown in the examples, our proposed approach is expected to reduce conservatism. 


\section{Robust controller design}

This section develops the problem of stabilization for closed-loop T-S fuzzy systems, such that the obtained controller will guarantee the asymptotical stability of the closed-loop system (8).

Theorem 2: For given scalars $\overline{\mathrm{h}}_{1} \geq 0, \overline{\mathrm{h}}_{2} \geq 0$, the closed-loop systems (8) satisfying condition (2) is robustly asymptotically stable with feedback gains $\mathrm{K}_{\mathrm{j}}=\mathrm{Y}_{\mathrm{j}} \mathrm{X}^{-\mathrm{T}}$ $(\mathrm{j}=1,2, \ldots, \mathrm{r})$, , if there exist symmetric positive definite matrices $\mathrm{X}, \tilde{\mathrm{Q}}_{1}, \tilde{\mathrm{Q}}_{2}, \tilde{\mathrm{Z}}_{1}, \tilde{\mathrm{Z}}_{2}$ with appropriate dimensions, and scalar $\varepsilon_{\mathrm{ij}}>0$ such that $\widetilde{\mathrm{Q}}_{1}-\widetilde{\mathrm{Q}}_{2} \geq 0$ and the following linear matrix inequalities (LMIs) hold for $\mathrm{i}, \mathrm{j}=1,2, \ldots, \mathrm{r}$.

$$
\tilde{\Sigma}_{\mathrm{ij}}=\left[\begin{array}{cccccc}
\tilde{\Sigma}_{11}^{\mathrm{ij}} & \tilde{\Sigma}_{12}^{\mathrm{ij}} & \tilde{\Sigma}_{13}^{\mathrm{ij}} & \tilde{\Sigma}_{14}^{\mathrm{ij}} & \tilde{\Sigma}_{15}^{\mathrm{ij}} & \tilde{\Sigma}_{16}^{\mathrm{ij}} \\
* & \tilde{\Sigma}_{22}^{\mathrm{ij}} & \tilde{\Sigma}_{23}^{\mathrm{ij}} & 0 & 0 & 0 \\
* & * & \tilde{\Sigma}_{33}^{\mathrm{ij}} & \tilde{\Sigma}_{34}^{\mathrm{ij}} & 0 & \tilde{\Sigma}_{36}^{\mathrm{ij}} \\
* & * & * & \tilde{\Sigma}_{44}^{\mathrm{ij}} & \tilde{\Sigma}_{45}^{\mathrm{ij}} & 0 \\
* & * & * & * & \tilde{\Sigma}_{55}^{\mathrm{ij}} & 0 \\
* & * & * & * & * & \tilde{\Sigma}_{55}^{\mathrm{ij}}
\end{array}\right]<0
$$

where,

$\tilde{\Sigma}_{11}^{\mathrm{ij}}=\mathrm{A}_{0 \mathrm{i}} \mathrm{X}+\mathrm{XA}_{0 \mathrm{i}}^{\mathrm{T}}+\mathrm{B}_{\mathrm{i}} \mathrm{Y}_{\mathrm{j}}+\mathrm{Y}_{\mathrm{j}}^{\mathrm{T}} \mathrm{B}_{\mathrm{j}}^{\mathrm{T}}-\tilde{\mathrm{Z}}_{1}+\tilde{\mathrm{Q}}_{1}$,

$\tilde{\Sigma}_{12}^{\mathrm{ij}}=\tilde{\mathrm{Z}}_{1}, \tilde{\Sigma}_{13}^{\mathrm{ij}}=\mathrm{A}_{\mathrm{di}} \mathrm{X}^{\mathrm{T}}, \tilde{\Sigma}_{14}^{\mathrm{ij}}=\mathrm{XA}_{0 \mathrm{i}}^{\mathrm{T}}+\mathrm{Y}_{\mathrm{j}}^{\mathrm{T}} \mathrm{B}_{\mathrm{i}}$,

$\tilde{\Sigma}_{15}^{\mathrm{ij}}=\mathrm{D}_{\mathrm{i}}, \tilde{\Sigma}_{16}^{\mathrm{ij}}=\varepsilon_{\mathrm{ij}}\left(\mathrm{XE}_{0 \mathrm{i}}^{\mathrm{T}}+\mathrm{Y}_{\mathrm{ij}}^{\mathrm{T}} \mathrm{E}_{\mathrm{bi}}^{\mathrm{T}}\right)$,

$\tilde{\Sigma}_{22}^{\mathrm{ij}}=-\tilde{\mathrm{Z}}_{1}-\tilde{\mathrm{Z}}_{2}-\left(1-\mathrm{d}_{1}\right)\left(\tilde{\mathrm{Q}}_{1}-\tilde{\mathrm{Q}}_{2}\right), \tilde{\Sigma}_{23}^{\mathrm{ij}}=\tilde{\mathrm{Z}}_{2}$

$\tilde{\Sigma}_{33}^{\mathrm{ij}}=-\tilde{Z}_{2}-\left(1-\mathrm{d}_{1}-\mathrm{d}_{2}\right) \tilde{\mathrm{Q}}_{2}, \quad \tilde{\Sigma}_{34}^{\mathrm{ij}}=\mathrm{XA}_{\mathrm{di}}^{\mathrm{T}}$,

$\tilde{\Sigma}_{35}^{\mathrm{ij}}=\varepsilon_{\mathrm{ij}} \mathrm{XE}_{\mathrm{di}}^{\mathrm{T}} \quad \tilde{\Sigma}_{44}^{\mathrm{ij}}=\mathrm{h}_{1}^{2} \tilde{\mathrm{Z}}_{1}+\mathrm{h}_{2}^{2} \tilde{\mathrm{Z}}_{2}-2 \mathrm{X}^{\mathrm{T}}, \tilde{\Sigma}_{45}^{\mathrm{ij}}=\mathrm{D}_{\mathrm{i}}$,

$\tilde{\Sigma}_{55}^{\mathrm{ij}}=-\varepsilon_{\mathrm{ij}} \mathrm{I}, \tilde{\Sigma}_{66}^{\mathrm{ij}}=-\varepsilon_{\mathrm{ij}} \mathrm{I}$

Proof: Pre- and post-multiply both sides of Eq. (9) with $\operatorname{diag}\left[\begin{array}{llllll}X & X & X & X & I & I\end{array}\right]$ and its transpose. Letting $\mathrm{X}=\mathrm{P}^{-1}, \mathrm{XZ}_{1} \mathrm{X}^{\mathrm{T}}=\tilde{\mathrm{Z}}_{1}, \mathrm{XZ}_{2} \mathrm{X}^{\mathrm{T}}=\tilde{\mathrm{Z}}_{2}, \mathrm{XQ}_{1} \mathrm{X}^{\mathrm{T}}=\tilde{\mathrm{Q}}_{1}$, $\mathrm{XQ}_{2} \mathrm{X}^{\mathrm{T}}=\tilde{\mathrm{Q}}_{2}$ and $Y_{j}=K_{j} X^{T}$ we arrive at (22) and the proof is complete.

\section{Numerical examples}

This section gives two numerical examples to demonstrate the effectiveness of the proposed approach by theorem 1 and theorem 2 .

Example 1: Consider a system with the following rules: [16, Example 1]

Rule 1: If $\mathrm{X}_{1}(\mathrm{t})$ is $\mathrm{W}_{1}$

Then $\dot{x}(t)=A_{01} x(t)+A_{d 1} x\left(t-h_{1}(t)-h_{2}(t)\right)$

Rule 2: If $\mathrm{x}_{2}(\mathrm{t})$ is $\mathrm{W}_{2}$

Then $\dot{x}(t)=A_{02} x(t)+A_{d 2} x\left(t-h_{1}(t)-h_{2}(t)\right)$

and the membership functions for rule 1 and rule 2 are $\mu_{1}(\mathrm{z}(\mathrm{t}))=\frac{1}{1+\exp \left(-2 \mathrm{x}_{1}(\mathrm{t})\right)}, \mu_{2}(\mathrm{z}(\mathrm{t}))=1-\mu_{1}(\mathrm{z}(\mathrm{t}))$

where,

$\mathrm{A}_{01}=\left[\begin{array}{cc}-2 & 0 \\ 0 & -0.9\end{array}\right], \mathrm{A}_{\mathrm{d} 1}=\left[\begin{array}{cc}-1 & 0 \\ -1 & -1\end{array}\right]$,

$\mathrm{A}_{02}=\left[\begin{array}{cc}-1 & 0.5 \\ 0 & -1\end{array}\right]$,

$\mathrm{A}_{\mathrm{d} 2}=\left[\begin{array}{cc}-1 & 0 \\ 0.1 & -1\end{array}\right]$

Our purpose is to calculate the upper bound $\bar{h}_{1}$ of $h_{1}(t)$, or $\bar{h}_{2}$ of $h_{2}(t)$, when the other is known. The maximum allowable upper bound of the system is obtained by adding $\bar{h}_{1}$ and $\bar{h}_{2}$, i.e., $\bar{h}=\bar{h}_{1}+\bar{h}_{2}$.

Table 1 lists the results of the maximum allowable delay bounds $\bar{h}=\bar{h}_{1}+\bar{h}_{2}$ derived from various methods including, Tian and Peng [18], Peng et al. [16], Fan et al. [23], Idrissi and Tissir [20] and the one proposed in this paper. Table 1 shows that the results obtained from our method are less conservative than those obtained from existing methods. Figure 1 shows the response of the fuzzy system with $\bar{h}=1.897$ and initial condition $x(t)=\left[\begin{array}{ll}1 & 0.2\end{array}\right]^{T}, t \in\left[\begin{array}{ll}-1.987 & 0\end{array}\right]$.

Now, let us consider the number of variables in different methods. Generally speaking, for the same upper delay bound $\bar{h}$, fewer variables require less computational power. While Idrissi and Tissir [20] required 27 variables in Theorum 1, our proposed approach requires only 15, and provides a better upper delay bound $\bar{h}$ with fewer variables as compared to 23 for Peng et al. [16] (Corollary 1) and 38 for Fan et al. [23].

The simulation result shows that system (Example 1 ) is asymptotically stable for the upper bound $\bar{h}=1.897$, see Fig. 1.

Example 2: Consider the following T-S fuzzy model with additive time delay [17,Example3]

Rule 1: If $\left(\mathrm{x}_{2}(\mathrm{t}) / 0.5\right)$ is about 0.5

Then

$\dot{\mathrm{x}}(\mathrm{t})=\left(\mathrm{A}_{01}+\Delta \mathrm{A}_{01}\right) \mathrm{x}(\mathrm{t})+\left(\mathrm{A}_{\mathrm{d} 1}+\Delta \mathrm{A}_{\mathrm{d} 1}\right) \mathrm{x}\left(\mathrm{t}-\mathrm{h}_{1}(\mathrm{t})-\mathrm{h}_{2}(\mathrm{t})\right)$ $+\mathrm{B}_{1} \mathrm{u}(\mathrm{t})$

Rule 2: If $\left(\mathrm{x}_{2}(\mathrm{t}) / 0.5\right)$ is about $\pi$ or $-\pi$ Then

$\dot{\mathrm{x}}(\mathrm{t})=\left(\mathrm{A}_{02}+\Delta \mathrm{A}_{02}\right) \mathrm{x}(\mathrm{t})+\left(\mathrm{A}_{\mathrm{d} 2}+\Delta \mathrm{A}_{\mathrm{d} 2}\right) \mathrm{x}\left(\mathrm{t}-\mathrm{h}_{1}(\mathrm{t})-\mathrm{h}_{2}(\mathrm{t})\right)$ $+\mathrm{B}_{2} \mathrm{u}(\mathrm{t})$

where,

$$
\begin{aligned}
& \mathrm{A}_{01}=\left[\begin{array}{cc}
-0 & 1 \\
0.1 & -2
\end{array}\right], \mathrm{A}_{02}=\left[\begin{array}{cc}
0 & 1 \\
0.1 & -0.5-1.5 \beta
\end{array}\right], \\
& \mathrm{B}_{1}=\mathrm{B}_{2}=\left[\begin{array}{l}
0 \\
1
\end{array}\right], \mathrm{A}_{\mathrm{d} 1}=\mathrm{A}_{\mathrm{d} 2}=\left[\begin{array}{cc}
0.1 & 0 \\
0.1 & -0.2
\end{array}\right], \beta=\frac{0.01}{\pi},
\end{aligned}
$$


$\mathrm{D}_{\mathrm{i}}=\left[\begin{array}{cc}-0.03 & 0 \\ 0 & 0.03\end{array}\right], \mathrm{E}_{0 \mathrm{i}}=\left[\begin{array}{cc}-0.15 & 0.2 \\ 0 & 0.04\end{array}\right]$,

$\mathrm{E}_{\mathrm{di}}=\left[\begin{array}{cc}-0.05 & -0.35 \\ 0.08 & -0.45\end{array}\right], \mathrm{i}=1,2$.

and $\beta$ is used to prevent system matrices from being singular.

The membership functions are set as follows:

$$
\begin{aligned}
& \mu_{1}(\mathrm{z}(\mathrm{t}))=\left(1-\frac{1}{1+\exp \left(-3\left(\left(\mathrm{x}_{2}(\mathrm{t}) / 0.5\right)-0.5 \pi\right)\right)}\right) \\
& \times\left(\frac{1}{1+\exp \left(-3\left(\left(\mathrm{x}_{2}(\mathrm{t}) / 0.5\right)+0.5 \pi\right)\right)}\right) \\
& \mu_{2}(\mathrm{z}(\mathrm{t}))=1-\mu_{1}(\mathrm{z}(\mathrm{t}))
\end{aligned}
$$

when $\mathrm{d}=0$, (e.g. $\mathrm{d}_{1}=0$ and $\mathrm{d}_{2}=0$ ). Table 2 lists the results using Theorem 2 of this paper, where $\Delta \mathrm{A}_{0 \mathrm{i}}=\Delta \mathrm{A}_{\mathrm{di}}=0$, and the obtained results improve on those from previous works $[17,19,24,25]$.

Table 3 lists the results from using Theorem 2 of this paper, when $\Delta \mathrm{A}_{0 \mathrm{i}} \neq 0$ and $\Delta \mathrm{A}_{\mathrm{di}} \neq 0$.
We can see from Tables 2 and 3 that our proposed method provides less conservative results and smaller feedback gains than those derived from other previous methods $[17,19,24,25]$.

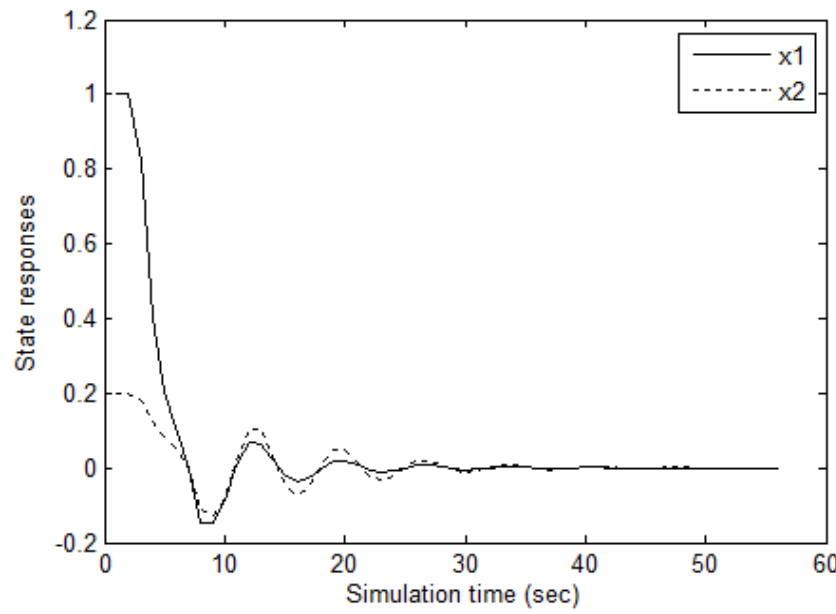

\begin{tabular}{|c|c|c|c|c|c|c|c|}
\hline Methods & \multicolumn{6}{|c|}{ Upper bound $\overline{\mathrm{h}}$} & Num.var \\
\hline Tian and Peng [18](Corollary 1) & \multicolumn{6}{|c|}{1.597} & 60 \\
\hline Peng et al. [16] (Corollary 1) & \multicolumn{6}{|c|}{1.597} & 23 \\
\hline Fan et al. [23] & \multicolumn{6}{|c|}{1.597} & 38 \\
\hline - & \multicolumn{3}{|c|}{ upper bound $\overline{\mathrm{h}}_{2}$ for given $\overline{\mathrm{h}}_{1}$} & \multicolumn{3}{|c|}{ upper bound $\overline{\mathrm{h}}_{1}$ for given $\overline{\mathrm{h}}_{2}$} & - \\
\hline \multirow{2}{*}{ Idrissi and Tissir [20] } & $\overline{\mathrm{h}}_{1}=1$ & $\overline{\mathrm{h}}_{1}=1,2$ & $\overline{\mathrm{h}}_{1}=1,5$ & $\overline{\mathrm{h}}_{2}=0,2$ & $\overline{\mathrm{h}}_{2}=0,3$ & $\overline{\mathrm{h}}_{2}=0,5$ & - \\
\hline & 0,897 & 0,667 & 0,208 & 1,504 & 1,449 & 1,323 & 27 \\
\hline Theorem 1 of this paper & 0,897 & 0,667 & 0,208 & 1,504 & 1,449 & 1,323 & 15 \\
\hline
\end{tabular}

Figure 1. System time response (Example 1)

Table 1. Calculated upper bound $\overline{\mathrm{h}}$ for different cases.

Table 2. Calculated upper bound $\overline{\mathrm{h}}$ and controller feedback gains without uncertainties (example 2).

\begin{tabular}{|c|c|c|c|}
\hline Methods & Upper bound $\overline{\mathrm{h}}$ & $\mathrm{K}_{1}$ & $\mathrm{~K}_{2}$ \\
\hline Lin et al [17] & 1.725 & {$\left[\begin{array}{ll}30.6571 & 10.0442\end{array}\right]$} & {$\left[\begin{array}{ll}30.6589 & 11.1803\end{array}\right]$} \\
\hline Guan and Chen [24] & 3.4385 & {$\left[\begin{array}{lll}-0.9390 & 0.3123\end{array}\right]$} & {$\left[\begin{array}{ll}-0.3277 & -0.3636\end{array}\right]$} \\
\hline Chen et al [25] & 25.7865 & {$\left[\begin{array}{ll}-1.2141 & 0.8750\end{array}\right]$} & {$\left[\begin{array}{ll}-1.2141 & -0.6202\end{array}\right]$} \\
\hline \multirow{3}{*}{ Li et al [19] } & 25.7865 & {$\left[\begin{array}{ll}-0.9318 & 0.1265\end{array}\right]$} & {$\left[\begin{array}{ll}-0.9318 & -1.3687\end{array}\right]$} \\
\hline \multirow{2}{*}{-} & 26.8617 & {$\left[\begin{array}{lll}-0.9211 & 0.1344\end{array}\right]$} & {$\left[\begin{array}{ll}-0.9211 & -1.3609\end{array}\right]$} \\
\hline \multirow{2}{*}{ Theorem 2 of this paper } & $\overline{\mathrm{h}}_{1}=20, \overline{\mathrm{h}}_{2}=6.8617$ & {$\left[\begin{array}{ll}-0.7328 & -0.9645\end{array}\right]$} & {$\left[\begin{array}{ll}-0.8061 & -1.0609\end{array}\right]$} \\
\cline { 2 - 5 } & $\overline{\mathrm{h}}_{1}=7.1254, \overline{\mathrm{h}}_{2}=22$ & {$\left[\begin{array}{lll}-0.7269 & -0.8968\end{array}\right]$} & {$\left[\begin{array}{ll}-0.7996 & -0.9865\end{array}\right]$} \\
\hline
\end{tabular}


Table 3. Calculated upper bound $\overline{\mathrm{h}}$ and controller feedback gains with uncertainties (Example 2).

\begin{tabular}{|c|c|c|c|}
\hline Methods & Upper bound $\overline{\mathrm{h}}$ & $\mathrm{K}_{1}$ & $\mathrm{~K}_{2}$ \\
\hline Chen et al [25] & 20.5640 & {$\left[\begin{array}{ll}-1.3987 & -0.6601\end{array}\right]$} & {$\left[\begin{array}{ll}-1.3991 & -2.1607\end{array}\right]$} \\
\hline \multirow{2}{*}{ Li et al [19] } & 20.5640 & {$\left[\begin{array}{ll}-1.3778 & -1.9868\end{array}\right]$} & {$\left[\begin{array}{ll}-1.3778 & -3.4820\end{array}\right]$} \\
& 22.4582 & {$\left[\begin{array}{ll}-1.4470 & -2.0154\end{array}\right]$} & {$\left[\begin{array}{ll}-1.4470 & -3.5106\end{array}\right]$} \\
\hline \multirow{2}{*}{-} & Upper bound $\overline{\mathrm{h}}=\overline{\mathrm{h}}_{1}+\overline{\mathrm{h}}_{2}$ & - & - \\
\hline \multirow{2}{*}{ Theorem 2 of this paper } & $\overline{\mathrm{h}}_{1}=18, \overline{\mathrm{h}}_{2}=4.4582$ & {$\left[\begin{array}{lll}-0.9449 & -1.2548\end{array}\right]$} & {$\left[\begin{array}{ll}-0.9348 & -1.2755\end{array}\right]$} \\
\cline { 2 - 5 } & $\overline{\mathrm{h}}_{1}=13, \overline{\mathrm{h}}_{2}=15.4263$ & {$\left[\begin{array}{ll}-0.9298 & -1.1811\end{array}\right]$} & {$\left[\begin{array}{ll}-0.9185 & -1.2058\end{array}\right]$} \\
\hline
\end{tabular}

\section{Conclusion}

A delay-dependent stability analysis and is presented to resolve controller design problems for uncertain T-S fuzzy systems with two additive time varying delays. The proposed LMIs are obtained using the Lyapunov Krasovskii functional method and improved Jensen's inequality. Moreover, the maximum allowable upper delay bound and the feedback controller gain can be simultaneously obtained by solving the LMI set. The reduced conservativeness of the results is shown by two numerical examples.

\section{References}

[1] T. Takagi and M. Sugeno, "Fuzzy identification of its applications to modeling and control," IEEE Transactions Systems, Man, and Cybernetics, vol. 15, no. 1, pp. 116-132, 1985. doi: 10.1109/TSMC.1985.6313399

[2] X.-D. Liu and Q.-L. Zhang, "New approaches to $H_{\infty}$ controller designs based on fuzzy observers for T-S fuzzy systems via LMI," Automatica, vol. 39, no. 9, pp. 1571-1582, 2003. doi: 10.1016/S0005-1098(03)00172-9

[3] Y. Zhang and A.-H. Pheng, "Stability of fuzzy systems with bounded uncertain delays," IEEE Transactions on Fuzzy Systems, vol. 10, no. 1, pp. 92-97, 2002. doi: $10.1109 / 91.983283$

[4] X.-D. Liu and Q.-L. Zhang, "Approaches to quadratic stability conditions and $H_{\infty}$ control designs for T-S fuzzy systems," IEEE Transactions on Fuzzy Systems, vol. 11, no. 6, pp. 830-839, 2003. doi: 10.1109/TFUZZ.2003.819834

[5] Y.-Y. Cao and P. M. Frank, "Stability analysis and synthesis of nonlinear time-delay systems via linear TakagiSugeno fuzzy models," Fuzzy Sets and Systems, vol. 124, no. 2, pp. 213-229, 2001. doi: $10.1016 /$ S0165-0114(00)00120-2
[6] K. Gu, V. L. Kharitonov, and J. Chen, Stability of Timedelay Systems, New York: Springer Science \& Business Media, 2003.

[7] X. Jiang and Q.-L. Han, "Robust $H_{\infty}$ control for uncertain Takagi-Sugeno fuzzy systems with interval time-varying delay," IEEE Transactions on Fuzzy Systems, vol. 15, no. 2, pp. 321-331, 2007. doi: 10.1109/TFUZZ.2006.878251

[8] C. Lien, K. Yu, W. Chen, Z. Wan, and Y. Chung, "Stability criteria for uncertain Takagi-Sugeno fuzzy systems with interval time-varying delay," IET Control Theory and Applications, vol. 1, no. 3, pp. 764-769, 2007. doi: $10.1049 /$ iet-cta:20060299

[9] C. Peng, D. Yue, and Y.-C. Tian, "New approach on robust delay-dependent control for uncertain T-S fuzzy systems with interval time-varying delay," IEEE Transactions on Fuzzy Systems, vol. 17, no. 4, pp. 890900, 2009.

doi: $10.1109 /$ TFUZZ.2008.926586

[10] D. Yue, Q.-L. Han, and J. Lam, “Network-based robust H1 control of systems with uncertainty," Automatica, vol. 41, no. 6, pp. 999-1007, 2006. doi: 10.1016/i.automatica.2004.12.011

[11] J. P. Hespanha, P. Naghshtabrizi, and Y. Xu, "A survey of recent results in networked control systems," Proceeding of the IEEE, vol. 95, no. 1, pp. 138-162, 2007. doi: $10.1109 /$ JPROC.2006.887288

[12] C. Peng and Y.-C. Tian, "State feedback controller design of networked control systems with interval time-varying delay and nonlinearity," International Journal of Robust and Nonlinear Control, vol. 18, no. 12, pp. 1285-1301, 2008.

doi: $10.1002 /$ rnc. 1278

[13] C.-H. Fang, Y.-S. Liu, S.-W. Kau, L. Hong, and C.-H. Lee, “A new LMI based approach to relaxed quadratic stabilization of T-S fuzzy control systems," IEEE 
Transactions on Fuzzy Systems, vol. 14, no.3, pp. 386397, 2006.

doi: 10.1109/TFUZZ.2006.876331

H.-N. Wu and H.-X. Li, "New approach to delaydependent stability analysis and stabilisation for continuous time fuzzy systems with time-varying delay," IEEE Transactions on Fuzzy Systems, vol. 15, no. 3, pp. 482-493, 2007.

doi: $10.1109 /$ TFUZZ.2006.889963

[14] J. Yoneyama, "Robust stability and stabilization for uncertain Takagi-Sugeno fuzzy time-delay systems," Fuzzy Sets and Systems, vol. 158, no. 2, pp. 115-134, 2007.

doi: $10.1016 /$ i.fss.2006.09.004

[15] C. Peng, Y.-C. Tian, and E.G. Tian, "Improved delaydependent robust stabilization conditions of uncertain T-S fuzzy systems with time-varying delay," Fuzzy Sets and Systems, vol. 159, no. 20, pp. 2713-2729, 2008. doi: $10.1016 /$ j.fss.2008.03.009

[16] C. Lin, Q.-G. Wang, and T.-H. Lee, "Delay-dependent LMI conditions for stability and stabilization of T-S fuzzy systems with bounded time-delay," Fuzzy Sets and Systems, vol. 157, no. 9, pp. 1229-1247, 2006. doi: $10.1016 /$ i.fss.2005.10.001

[17] E.G. Tian and C. Peng, "Delay dependent stability analysis and synthesis of uncertain T-S fuzzy systems with time-varying delay," Fuzzy Sets and Systems, vol. 157, no. 4, pp. 544-559, 2006. doi: $10.1016 /$ i.fss.2005.06.022

[18] L. Li, X. Liu, and T. Chai, "New approaches on $H_{\infty}$ control of T-S fuzzy systems with interval time-varying delay,"
Fuzzy Sets and Systems, vol. 160, no. 12, pp. 1669-1688, 2009.

doi: 10.1016/j.fss.2008.11.021

[19] S. Idrissiand and E. H. Tissir, "Delay Dependent Robust Stability of T-S Fuzzy Systems with Additive Time Varying Delays," Applied Mathematical Sciences, vol. 6, no. 1, pp. 1-12, 2012.

[20] I. R. Peterson and C. V. Hollot, "A Riccati equation approach to the stabilization of uncertain linear systems," Automatica, vol. 22, no. 4, pp. 397-411, 1986. doi: 10.1016/0005-1098(86)90045-2

[21] R. Dey, G. Ray, S. Ghosh, and A. Rakshit, "Stability analysis for continuous system with additive timevarying delays: A less conservative result," Applied Mathematics and Computation, vol. 215, no. 10, pp. 3740-3745, 2010.

[22] F. Liu, M. Wu, Y. He, and R. Yokoyama, "New delaydependent stability criteria for T-S fuzzy systems with time-varying delay," Fuzzy Sets and Systems, vol. 161, no. 15, pp. 2033-2042, 2010. doi: 10.3182/20080706-5-KR-1001.00043

[23] X.-P. Guan and C.-L. Chen, “Delay-dependent guaranteed cost control for T-S fuzzy systems with time delays," IEEE Transactions on Fuzzy Systems, vol. 12, no. 2, pp. 236-249, 2004. doi: $10.1109 /$ TFUZZ.2004.825085

[24] B. Chen, X.-P. Liu, and S.-C Tong, "New delay-dependent stabilization conditions of T-S fuzzy systems with constant delay," Fuzzy Sets and Systems, vol. 158, no. 20, pp. 2209-2224, 2007. doi: $\underline{10.1016 / \text { i.fss.2007.02.018 }}$ 